\title{
Listeriosis en bovinos de la Provincia de Buenos Aires, Argentina
}

\author{
Margineda, C.A.; Cantón, G.; Lischinsky, L.; Moreira, A.; Campero, C.M. \\ Grupo de Sanidad Animal, Instituto Nacional de Tecnología Agropecuaria (INTA) \\ Balcarce, EEA Balcarce, Ruta Nacional 226, km 73,5 (7620) Buenos Aires, \\ Argentina. Tel/fax: 54-02266-439100/01. E-mail: carlosmargineda@gmail.com.
}

\begin{abstract}
Resumen
Margineda, C.A.; Cantón, G.; Lischinsky, L.; Moreira, A.; Campero, C.M.: Listeriosis en bovinos de la Provincia de Buenos Aires, Argentina. Rev. vet. 23: 1, 32-37, 2012. Se realizó un estudio retrospectivo de casos de listeriosis bovina ocurridos en diferentes partidos de Provincia de Buenos Aires (Argentina). Se utilizaron las técnicas diagnósticas de histopatología, bacteriología e inmunohistoquímica. De 17 casos confirmados por esta última técnica, en 7 de ellos se dispuso de material para el cultivo bacteriológico y solo en 3 casos se pudo aislar Listeria monocytogenes. El 64,7\% de los casos de listeriosis se presentaron en rodeos que consumían ensilado de maíz. La enfermedad se diagnosticó con mayor frecuencia en sistemas de producción intensivos (feedlot y tambos). Cuatro casos negativos al cultivo de $L$. monocytogenes se lograron confirmar por inmunohistoquímica, por lo cual esta técnica se revela valiosa dado que su empleo mejora la eficiencia diagnóstica de los casos de listeriosis bovina.
\end{abstract}

Palabras claves: bovino, Listeria monocytogenes, inmunohistoquímica, Argentina.

\begin{abstract}
Margineda, C.A.; Cantón, G.; Lischinsky, L.; Moreira, A.; Campero, C.M.: Bovine listeriosis in Buenos Aires Province, Argentina. Rev. vet. 23: 1, 32-37, 2012. A retrospective study of bovine listeriosis cases occurring in different counties of Buenos Aires Province (Argentina) was made. Diagnosis was performed by histopathology, bacteriology and immunohistochemistry. From 17 positive cases confirmed by immunohistochemistry, samples were available only in 7 for bacteriological study, and Listeria monocytogenes was isolated only in 3 of them. Of all the cases, $64.7 \%$ were associated with the consumption of corn silage. The disease was most frequently diagnosed in intensive production systems, such as feedlot and dairy herds. One of the isolated strains of L. monocytogenes was resistant to rifampicin and sulfonamides. After immunohistochemistry, L. monocytogenes positive labeling was observed in 4 cases with previous negative bacteriological isolation. Immunohistochemistry proved to be a valuable technique and improved the diagnosis of bovine listeriosis.
\end{abstract}

Key words: cattle, Listeria monocytogenes, immunohistochemistry, Argentina.

\section{INTRODUCCIÓN}

La listeriosis bovina es una enfermedad infecciosa producida por Listeria monocytogenes ${ }^{5,19}$. Esta bacteria es ubicua, se la ha aislado de suelo, pasturas, ensilados de maíz, aguas residuales y materia fecal de animales domésticos ${ }^{5,30}$. Aunque $L$. monocytogenes es capaz de infectar a una gran variedad de especies animales, la infección ocurre primariamente en rumiantes domésticos y seres humanos.

Proyecto INTA AESA 203981, Monitoreo y vigilancia epidemiológica de las enfermedades animales.

Recibido: 5 diciembre 2011 / Aceptado: 8 febrero 2012
En bovinos, existen diferentes presentaciones clínicas causadas por L. monocytogenes: (A) septicémica con abscesos en vísceras como hígado, bazo y linfonódulos, (B) abortos, (C) encefalitis, (D) queratoconjuntivitis y uveítis, (E) mastitis ${ }^{5,11,12,20,23,25,28}$. La forma septicémica suele afectar a animales recién nacidos y la forma nerviosa es más común en bovinos y ovinos adultos ${ }^{3,16,20,23}$. En rumiantes, la presentación nerviosa ocurre en forma esporádica o como brotes. Los casos generalmente están asociados al consumo de silajes, ya sea mal elaborados o acondicionados en forma deficiente $^{5,20,26}$. Si el ensilado no está bien confeccionado el $\mathrm{pH}$ suele elevarse a valores superiores a 5, generando condiciones ideales para la multiplicación bacteriana 19,20 . En las necropsia de casos de encefalitis general- 
mente no se observan lesiones macroscópicas en el sistema nervioso central ${ }^{2,14,27}$. Las lesiones microscópicas se confinan al tronco encefálico y consisten en infiltrado de células mononucleares alrededor de los vasos sanguíneos, microabscesos acompañados de focos de malacia, células de Gitter y meningoencefalitis mononuclear en el área basal del encéfalo 2, 14, 22, 23, 27 . Los signos clínicos de la forma encefálica de listeriosis comprenden: depresión o excitabilidad, anorexia y otros signos relacionados a la disfunción de los nervios craneanos. Los signos nerviosos suelen ser asimétricos, con ptosis mandibular, estrabismo, ptosis del pabellón auricular, torsión de la cabeza, marcha en círculos o tambaleante, ptosis palpebral, parálisis facial, respiración estertorosa, disfagia, parálisis de la lengua, nistagmos, decúbito permanente y pedaleo ${ }^{2,20,26,27}$.

El diagnóstico presuntivo de la enfermedad se establece por las lesiones histológicas características y se confirma por el aislamiento bacteriano, el cual puede ser laborioso ${ }^{14}$. La inmunohistoquímica (IHQ) es una técnica rápida y sensible que permite confirmar el diagnóstico de listeriosis en diferentes tejidos cuando los aislamientos son negativos, o no se dispone de tejidos frescos para realizarlo $3,6,9,10,13,29$.

El objetivo de este trabajo fue realizar un estudio descriptivo de los hallazgos clínicos, bacteriológicos y patológicos de 17 casos de listeriosis bovina diagnosticados por el Servicio de Diagnóstico Veterinario Especializado (SDVE) del Instituto Nacional de Tecnología Agropecuaria (INTA, Balcarce, Argentina) durante los años 2002-2010.

\section{MATERIAL Y MÉTODOS}

Antecedentes. Se realizó un estudio retrospectivo de los casos de listeriosis con diagnóstico confirmado por cultivos y/o IHQ en muestras de tejidos de bovinos de rodeos comerciales. Se recolectó información anamnésica de los registros de cada caso sobre el tipo de alimentación, signos clínicos, edad, raza, sistema de producción e indicadores epidemiológicos, cuando estuvo disponible.

Examen patológico. Se obtuvieron muestras de cerebro de animales muertos espontáneamente con signos clínicos nerviosos y un feto abortado. Las mismas se fijaron durante 4 días en formol bufferado al $10 \%$. Para el análisis histopatológico se seleccionaron diferentes sectores del encéfalo: corteza frontal, corteza occipital, cerebelo, tálamo, tubérculos cuadrigéminos, protuberancia, pedúnculos y médula oblonga. Del feto se obtuvieron muestras de corazón, pulmón, lengua, abomaso, intestino delgado y grueso, glándula adrenal, hígado, bazo, riñón, músculo esquelético y piel. Las muestras fueron deshidratadas e incluídas en bloques de parafina. Las secciones fueron cortadas a 4-5 $\mu \mathrm{m}$ y teñidas con hematoxilina-eosina (H\&E).

Inmunohistoquímica. Los tejidos que presentaron lesiones compatibles con las producidas por L. monocytogenes fueron procesadas mediante IHQ ${ }^{6,12}$. Dichos tejidos fueron cortados a 4-5 $\mu$ m espesor y se montaron en histoslides (Micro-Probe Staining System, Fisher Scientific, Pittsburgh, USA). Las secciones fueron procesadas por la técnica de Avidina Biotina Peroxidasa (ABC, Vector Lab, USA) empleando un anticuerpo primario contra L. monocytogenes producido en conejo y diluido 1:1500 (Listeria O antiserum Poly Difco 230250-0, Difco Lab., USA). Se emplearon tejidos controles positivos y negativos existentes en el Laboratorio de Patología Veterinaria del INTA Balcarce.

Procedimientos bacteriológicos. En los casos de encefalitis los cultivos bacterianos se realizaron a partir de macerados de médula oblonga cultivados en agar sangre Columbia a $37^{\circ} \mathrm{C}$ en aerobiosis. Las muestras negativas a dichos cultivos fueron reprocesadas: luego de 15 días de enriquecimiento en frío a $4^{\circ} \mathrm{C}$ se volvieron a cultivar en agar sangre Columbia como se mencionó. En el caso del feto abortado (caso $\mathrm{N}^{\circ} 10$ ) se recolectaron muestras de líquido de abomaso, placenta y pulmón. Las mismas se sembraron en agar sangre Columbia y agar Mac Conkey e incubaron a $37^{\circ} \mathrm{C}$ en aerobiosis. Los cultivos fueron confirmados como $L$. monocytogenes utilizando pruebas bioquímicas convencionales ${ }^{19}$.

Otras técnicas. Con el objetivo de descartar la coexistencia de distinta/s patología/s abortiva/s, sobre el feto se utilizaron otras técnicas diagnósticas complementarias descriptas previamente ${ }^{4}$. Se tomaron muestras de pulmón y líquido abomasal para realizar cultivo bacteriológico (aeróbicos y microaerófilos), cultivo y test de inmunofluorescencia directa para la detección de Campylobacter fetus. Además, el fluido abomasal se sembró en medio de cultivo para Tritrichomonas foetus. Los tejidos fetales se procesaron además con la técnica de IHQ para la detección de Neospora caninum ${ }^{4}$. Se obtuvieron muestras de bazo para cultivo viral, las cuales fueron suspendidas en medio de Hank's, homogeneizadas y centrifugadas. Se inocularon alícuotas del sobrenadante en células Madin-Darby Bovine Kidney (MDBK), realizándose 4 pasajes por cultivos celulares de 72 h cada uno ${ }^{4}$.

\section{RESULTADOS}

Antecedentes. Luego de analizar los casos recibidos durante el periodo 2002-2010 se identificaron 17 casos de listeriosis confirmados mediante IHQ, de los cuales 16 estaban asociados a encefalitis y uno fue un feto abortado de 4 meses de gestación (Tablas 1 y 2). Los casos ocurrieron en diferentes partidos de la Provincia de Buenos Aires: Bahía Blanca $(n=1)$, Balcarce $(n=3)$, Bolívar $(n=2)$, Chivilcoy $(n=1)$, General Pueyrredón $(n=1)$, General Villegas $(n=1)$, Navarro $(n=2)$, Necochea $(n=1)$, Pehuajó $(n=1)$, Partido de la Costa $(n=1)$, Tandil $(n=1)$ y Trenque Lauquen $(n=1)$. Los parámetros epidemiológicos estuvieron disponibles en solo 3 casos $\left(\mathrm{N}^{\mathrm{o}} 14,15\right.$ y 16), registrando una morbilidad de entre 0,01 y 7,1\% y una letalidad de entre 87 y 100\%. En 3 casos $\left(\mathrm{N}^{\circ} 4,6\right.$ y 16) los animales afectados habían sido 
Tabla 1. Caracterización epidemiológica de los casos de listeriosis bovina.

\begin{tabular}{lcccc}
\hline caso & categoría/raza/edad & mes & aptitud & alimentación \\
\hline \multicolumn{2}{l}{ animales $>$ de 24 meses } & & & \\
1 & Vaca / HA / 4 A & 7 & lechera & S de maíz + algodón + V avena. \\
2 & Vaca / HA / 3,5 A & 10 & lechera & S de maíz. \\
3 & Vaca / HA / Sd & 9 & lechera & S de maíz. \\
4 & Vaca / HA / 4 A & 5 & lechera & S de maíz. \\
5 & Vaca / HA / Sd & 7 & lechera & S de maíz. \\
6 & Toro / AA / 3 A & 8 & cría & pastura natural. \\
7 & Vaca / HA / Sd & 11 & lechera & S de maíz. \\
8 & Vaca / HA / Sd & 2 & lechera & S de pastura + S de maíz. \\
9 & Vaca / HA / 3,5 A & 8 & lechera & S de maíz. \\
animales $<$ de 24 meses & & & \\
10 & feto / AA / 4 M & 6 & cría & campo natural. \\
11 & novillo / Sd / 12 M & 5 & feedlot & G de maíz + expeller girasol + N. \\
12 & novillo / Sd / 16 M & 3 & feedlot & G de maíz + expeller girasol + N. \\
13 & novillo / Sd / 18 M & 8 & feedlot & S de maíz + expeller girasol + N. \\
14 & novillo / Sd / 14 M & 8 & feedlot & G de maíz + expeller girasol + N. \\
15 & novillo / Sd / Sd & 6 & feedlot & S de maíz + expeller girasol + N. \\
16 & novillo / Sd / 18 M & 9 & feedlot & S de maíz + pasturas. \\
17 & novillo / Sd / 14 M & 6 & invernada pastoril & pastura natural. \\
\hline
\end{tabular}

Sd: sin dato; HA: Holando Argentino; AA: Aberdeen Angus; M: meses; A: años; V: verdeo; S: silaje, G: granos enteros, N: núcleo vitamínico-mineral.

En el feto abortado se registró miocarditis multifocal necrotizante difusa con infiltrado inflamatorio mononuclear. La IHQ para detección de $N$. caninum resultó negativa. En la Tabla 2 se detallan los hallazgos de los casos presentados.

Inmunohistoquímica. En los 17 casos se identificaron antígenos de L. monocytogenes siendo la inmunotinción más notoria a nivel de los microabscesos y en los focos de necrosis presentes en el tejido nervioso. En el feto abortado, la IHQ resultó positiva en corazón, pulmón, hígado, intestino delgado y grueso. L. monocytogenes se observó como pequeños gránulos ovoides o circulares de color rojizo, en el citoplasma de fagocitos y neutrófilos y como pequeñas estructuras cocobacilares extracelulares. Ocasionalmente, se observaron dichos antígenos en los

tratados previamente con penicilina por vía parenteral. En 11/17 de los casos estudiados (64,7\%), el silaje de maíz fue utilizado como suplemento nutricional (Tabla 1). Se observó una mayor presentación de casos clínicos durante los meses de invierno y principio de primavera (Figura 1). El feto pertenecía a un rodeo de 510 vacas Aberdeen Angus en pastoreo sin suplementación (fue el único aborto registrado). En la Tabla 2 se mencionan los signos clínicos disponibles; los más frecuentes fueron ataxia/incoordinación $(9 / 14)$ y marcha en círculo y depresión (6/14).

Examen patológico. Al examen macroscópico del SNC de los casos de encefalitis, solamente en uno se observó hiperemia en meninges y múltiples focos de reblandecimiento en la médula oblonga de $0,5 \mathrm{~cm}$ de diámetro (caso $\mathrm{N}^{\circ} 17$ ). A la necropsia del feto no se observaron lesiones macroscópicas.

Al examen histopatológico de los casos de encefalitis, se observaron múltiples focos de necrosis acompañada de abundante cantidad de neutrófilos (microabscesos) e infiltrado celular mononuclear (predominantemente macrófagos y escasos linfocitos) a nivel del tronco encefálico, tanto en la sustancia gris como en la blanca. Los mismos fueron más severos en la protuberancia y bulbo raquídeo. También se detectaron manguitos perivasculares con infiltrado de células mononucleares (básicamente macrófagos, linfocitos y ocasionales plasmocitos) especialmente en los vasos sanguíneos adyacentes a los focos de necrosis presentes del tejido nervioso. En el caso $\mathrm{N}^{\circ} 14$ también se observaron lesiones en corteza cerebral, caracterizadas por meningitis mononuclear y manguitos perivasculares con predominio de macrófagos y linfocitos. sitios de infiltrado perivascular en el SNC. Tanto los tejidos controles positivos como los negativos se comportaron adecuadamente a la IHQ.

Procedimientos bacteriológicos. En 7/17 casos se pudo realizar el cultivo bacteriológico obteniéndose $L$. monocytogenes en 3 oportunidades. Del feto abortado, dicho agente fue aislado del líquido de abomaso, pulmón y placenta. En los 10 casos restantes, los tejidos no se pudieron cultivar por haberse recibido únicamente órganos formolados. De los 3 aislamientos positivos (casos $\mathrm{N}^{\circ} 2,10$ y 16), las colonias desarrollaron a las $24 \mathrm{~h}$ de incubación en pureza siendo todas ellas $\beta$-hemolíticas. A la microscopía, se observaron bacilos Gram positivos, clasificados bioquímicamente como L. monocytogenes. Las muestras de enriquecimiento en frío y resembradas fueron negativas (casos $\mathrm{N}^{\circ} 1,11$, 13 y 17). No se aislaron otros microorganismos en las muestras del SNC.

Otras técnicas. Las muestras extraídas del feto fueron negativas al aislamiento viral y las pruebas para diagnóstico de trichomonosis, campylobacteriosis, brucelosis y neosporosis.

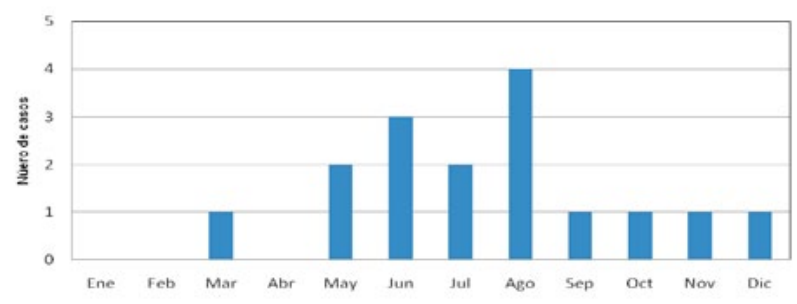

Figura 1. Casos de listeriosis en bovinos durante el periodo 2002-2010. 
Tabla 2. Aislamientos, hallazgos patológicos y signos clínicos de los casos estudiados.

\begin{tabular}{llll}
\hline caso & aislamiento & histopatología & signos clínicos \\
\hline animales $>$ de 24 m & & \\
1 & $(-)$ & MEC, MA, IPM, E, FN & decúbito lateral, pedaleo, fiebre. \\
2 & $(+)$ & MEC, MA, E, FN & ataxia, marcha en círculos. \\
3 & NR & MEC, IPM, E, FN & ataxia, ceguera, marcha en círculos, temblores musculares, babeo. \\
4 & NR & MEC, MA, E, FN & ataxia. \\
5 & NR & MEC, MA, IPM, E, FN & sialorrea, ptosis de labio inferior, dificultad para comer, pérdida de estado \\
& corporal. \\
6 & NR & MEC, MA, IPM, E, FN & depresión, marcha en círculos, decúbito lateral y pedaleo. \\
7 & NR & MA, IPM, E, FN & ataxia, ceguera, depresión. \\
8 & NR & MEC, IPM, E, FN & depresión, incoordinación. \\
9 & NR & MEC, MA, IPM, E, FN & depresión y marcha en círculos. \\
animales & de 24 & m & \\
10 & $(+)$ & Miocarditis mononuclear & feto abortado \\
11 & $(-)$ & MEC, MA, IPM, E, FN & depresión, incoordinación. \\
12 & NR & MEC, IPM, E, FN & Sd \\
13 & $(-)$ & MEC, MA, IPM, E, FN & incoordinación, ceguera, decúbito esternal, opistótonos. \\
14 & NR & MEC, MA, IPM, E, FN & marcha en círculos, incoordinación, bruxismo, ptosis auricular. \\
15 & NR & MEC, MA, IPM, E, FN & depresión, decúbito esternal y pedaleo. \\
16 & $(+)$ & MEC, IPM, E, FN & marcha en círculos, estrabismo, caídas, opistótonos. \\
$\mathbf{1 7}$ & $(-)$ & MEC, MA, IPM, E, FN & Depresión, marcha tambaleante incoordinación, decúbito lateral y pedaleo. \\
\hline
\end{tabular}

(+): positivo a L. monocytogenes; (-): negativo; NR: no realizado; Sd: sin datos; MEC: meningoencefalitis; MA: microabscesos; IPM: infiltrado perivascular mononuclear; E: encefalitis; FN: focos de necrosis.

\section{DISCUSIÓN}

En el presente estudio, el 64,7\% de los casos de listeriosis estuvieron asociados al consumo de silaje de maíz. Este resultado es coincidente con trabajos previos en similar zona geográfica ${ }^{3}$ y también con los hallazgos de otros autores ${ }^{5,12,20,26}$. Sin embargo, la asociación con el consumo de silaje no se observó en otros episodios registrados en rumiantes de Brasil y Cuba ${ }^{18,}$ 22, 23. Desafortunadamente, en este trabajo no se dispuso de información referente a la calidad de los silajes consumidos, tipo de construcciones (silo torta, trinchera, puente), condiciones de su elaboración y mantenimiento, así como la posibilidad de realizar cultivos y aislamientos en las muestras de silaje. La morbilidad de los brotes de encefalitis que se incluyen en este trabajo está comprendida dentro de los rangos mencionados por la bibliografía ${ }^{3,12,20,26}$.

Es importante mencionar que la enfermedad también ocurrió en animales mantenidos exclusivamente a campo en pasturas, sin suplementación (casos $\mathrm{N}^{\mathrm{o}} 6,10$ y 17) y se presentó con las características de un brote en animales de varios feedlots que no suplementaban con silo de maíz (casos $N^{\circ} 11,12$ y 13). Varios factores ambientales pueden causar un descenso de la resistencia a la infección por L. monocytogenes ${ }^{20}$. En los feedlots de nuestro país, se observan limitadas condiciones de cuidado que afectan el bienestar animal (exceso de barro, largos periodos de transporte, escasa sombra y reparos) las cuales, asociadas al estrés con que suelen ingresar los animales al encierre, crean condiciones predisponentes para la presentación de esta enfermedad ${ }^{15,20,21,26}$.
En este trabajo, al igual que lo mencionado para otras áreas geográficas 1,5,20,30, se observó estacionalidad de los casos con predominio en invierno y principio de primavera. Este hallazgo posiblemente esté ligado a un mayor estrés de los animales por los fríos y bajas temperaturas y/o por una mayor utilización del silaje durante el periodo invernal. El rango etario de los casos de encefalitis observados en el presente estudio (1 a 4 años de edad) es coincidente con el mencionado por otros autores ${ }^{1}$. Este hallazgo sugiere que los animales jóvenes tendrían mayor riesgo de adquirir la enfermedad posiblemente por efecto de los cambios de dentición asociados, los cuales facilitarían el ingreso de L. monocytogenes al SNC.

L. monocytogenes puede ingresar por vía hematógena y de allí pasar al SNC, cuando bacterias circulantes en el torrente sanguíneo invaden directamente células endoteliales de la barrera hematoencefálica; o bien mediante el transporte de bacterias hacia el SNC dentro de leucocitos circulantes en un mecanismo conocido como 'Caballo de Troya'. Además, la vía neurogénica ascendente es comúnmente descripta, donde las bacterias alcanzan el SNC desde tejidos periféricos por transporte intra-axonal ${ }^{7}$ siendo esta vía neurogénica la más común en rumiantes para invadir el SNC ${ }^{17}$. La encefalitis a nivel del tronco encefálico, suele producirse por este último mecanismo, por el cual la infección asciende a lo largo del nervio trigémino luego de producirse lesiones erosivas en la mucosa de la cavidad nasal u oral y el cambio de dientes en animales jóvenes. Estos cambios facilitan el ingreso bacteriano, luego se 
produce el ascenso por el nervio trigémino y así se establece la encefalitis basal ${ }^{5,21}$.

Los hallazgos macroscópicos en el SNC no suelen ser frecuentes ${ }^{2,14}$ habiéndose encontrado un solo caso $\left(\mathrm{N}^{\mathrm{o}} 17\right)$. Las alteraciones histológicas observadas estuvieron localizadas en el tronco encefálico (bulbo, puente y mesencéfalo) y son coincidentes con lo mencionado por otros autores 2, 10, 12, 14, 27. En el presente estudio, los antígenos de L. monocytogenes detectados por IHC fueron más frecuentes en las áreas de necrosis y microabscesos del SNC en coincidencia con otros trabajos 3, 13, 22, 23, 29 .

En todos los aislamientos de L. monocytogenes, las colonias desarrollaron a las $24 \mathrm{~h}$ de incubación. Los cultivos negativos lo fueron a pesar del método de enriquecimiento utilizado. Se deberían considerar otras técnicas de enriquecimiento o bien técnicas de biología molecular para disponer de otras herramientas diagnósticas. Es importante destacar lo ocurrido en el caso $\mathrm{N}^{\circ} 16$, que a pesar de que el animal fue tratado con penicilina durante 4 días, los cultivos bacteriológicos resultaron positivos.

L. monocytogenes puede tener predilección por la interfase materno-fetal placentaria ${ }^{5}$. En el presente estudio se registró un caso $\left(\mathrm{N}^{\circ} 10\right)$ de aborto en la gestación temprana, lo que no es coincidente con lo reportado por otros autores que mencionan mayor frecuencia de abortos en el último tercio gestacional ${ }^{5,17}$. En otros países $L$. monocytogenes es comúnmente mencionada como causa de aborto esporádico en el ganado bovino ${ }^{11}$. En Argentina no es frecuente su presencia como causa de abortos en el bovino ${ }^{4,8}$ o bien estar involucrada excepcionalmente en casos de muerte perinatal en bovinos ${ }^{16}$. En base a estos trabajos y nuestros hallazgos se puede aseverar que L. monocytogenes es solo un agente esporádico de pérdidas reproductivas en bovinos de nuestro medio.

La importancia de estos casos de listeriosis ocurridos en nuestro país, destaca el papel de los bovinos como posibles reservorios de cepas patógenas para la población humana. Los rumiantes, particularmente los bovinos sanos o enfermos, pueden contribuir a la diseminación de L. monocytogenes en el ambiente de los establecimientos ganaderos ${ }^{17}$. La leche puede contener L. monocytogenes como consecuencia de la contaminación exógena, especialmente en aquellos establecimientos que ordeñan en malas condiciones higiénicas ${ }^{24}$. Se deberían realizar nuevos estudios en Argentina, con el fin detectar L. monocytogenes en leche y así dilucidar cuál es su rol en la transmisión de la bacteria al hombre.

La técnica de IHQ utilizada en este trabajo representa una herramienta auxiliar que mejora significativamente el diagnóstico de la listeriosis bovina. Ello resulta particularmente relevante en casos dónde sólo se dispone de tejidos formolados, no pudiéndose realizar cultivos microbiológicos, o bien en animales que previamente recibieron tratamientos con antibióticos, lo cual dificulta la posibilidad del aislamiento. A pesar de que el cultivo se mantiene como un criterio importante para el diagnóstico de listeriosis, el uso de la histopatología e IHQ combinados mejoran la sensibilidad del diagnóstico.

En conclusión, surge que en la zona estudiada, la listeriosis bovina es más frecuente durante el invierno y en sistemas de producción intensivos (feedlot y tambos), donde se utilizan comúnmente diferentes suplementos alimenticios. La técnica de IHQ es útil para el diagnóstico de listeriosis, tanto para casos de encefalitis o abortos producidos por L. monocytogenes. En nuestro país, la forma encefálica debe incluirse en el diagnóstico diferencial de aquellas enfermedades que cursan con signos neurológicos en el ganado bovino.

Agradecimientos. Por su valiosa colaboración, a los miembros del Grupo de Sanidad Animal de INTA Balcarce.

\section{REFERENCIAS}

1. Barlow RM, McGorum B. 1985. Ovine listerial encephalitis: analysis, hypothesis and synthesis. Vet Rec 116: 233236.

2. Barros CS, Driemeier D, Dutra I, Lemos RA. 2006. Doenças do sistema nervoso de bovinos no Brasil. $1^{\text {a }} \mathrm{Ed}$, AGNS ed., Montes Claros, MG (Brasil) p. 67-70.

3. Campero CM, Odeón AC, Cipolla AL, Moore DP, Poso MA, Odriozola E. 2002. Demonstration of Listeria monocytogenes by immunohistochemistry in formalin-fixed brain tissues from natural cases of ovine and bovine encephalitis. J Vet Med B 49: 379-383.

4. Campero CM, Moore DP, Odeón A, Cipolla AL, Odriozola E. 2003. Aetiology of bovine abortion in Argentina. Vet Res Comm 27: 359-369.

5. Czuprynski CJ, Kathariou S, Poulsen K. 2010. Listeria. In: Pathogenesis of bacterial infections in animals (Gyles CL, Prescott JF, Songer GJ, Thoen CO, Ed), $4^{\text {th }}$ ed., Ames, Iowa (USA), p. 167-187.

6. Domingo M, Ramos JA, Dominguez L, Ferrer L, Marco A. 1986. Demonstration of Listeria monocytogenes with the PAP technique in formalin fixed and paraffin embedded tissues of experimental infected mice. $J$ Vet Med B 33: 537-542.

7. Drevets DA, Leenen PJ, Greenfield RA. 2004. Invasion of the central nervous system by intracellular bacteria. Clin Microbiol Rev 17: 323-347.

8. Fernandez ME, Campero C, Morrell E, Cantón GJ, Moore DP, Cano A, Malena R, Odeón AC, Paolicchi F, Odriozola ER. 2007. Pérdidas reproductivas en bovinos causadas por abortos, muertes prematuras, natimortos y neonatos: casuística del período 2006-2007. Rev Med Vet (Buenos Aires), 88: 246-254.

9. Hsu SM, Raine L, Fanger H. 1981. The use of avidin biotin peroxidase complex (ABC) in immunoperoxidase techniques: a comparison between $\mathrm{ABC}$ and unlabeled antibody (PAP) procedures. J Histochem Cytochem 29: 577-580. 
10. Johnson GC, Fales WH, Maddox CW, Ramos-Vara JA. 1995. Evaluation of laboratory tests for confirming the diagnosis of encephalitic listeriosis in ruminants. $J$ Vet Diagn Invest 7: 223-228.

11. Kirkbride C. 1993. Bacterial agents detected in a 10-year study of bovine abortions and stillbirths. J Vet Diagn Invest 5: 64-68.

12. Ladds PW, Dennis SM, Njoku CO. 1974. Pathology of listeric infection in domestic animals. Vet Bull 44: 67-74.

13. Marco A, Ramos JA, Dominguez J, Domingo M, Gonzalez L. 1988. Immunocytochemical detection of Listeria monocytogenes in tissue with the peroxidase-antiperoxidase technique. Vet Pathol 25: 385-387.

14. Maxie MG, Youssef S. 2007. Nervous system. In: Jubb, Kennedy, and Palmer's. Pathology of domestic animals (Maxie MG Ed.), $5^{\text {th }}$ ed, Saunders, Philadelphia, p. 405408.

15. Meredith CD, Schneider DJ. 1984. An outbreak of ovine listeriosis associated with poor flock management practices. J S Afric Vet Ass 55: 55-56.

16. Morrell EL, Moore DP, Odeón AC, Poso MA, Odriozola E, Cantón G, Paolicchi F, Malena R, Leunda MR, Morsella C, Campero CM. 2008. Retrospective study of bovine neonatal mortality: cases reported from INTA Balcarce, Argentina. Rev Arg Microbiol 40: 151-157.

17. Oevermann A, Zurbriggen A, Vandevelde M. 2010. Rhombencephalitis caused by Listeria monocytogenes in humans and ruminants: a zoonosis on the rise? Interdisc Persp Infect Dis 2010: 1-22 (Article ID 632513, doi:10.1155/2010/632513).

18. Pérez IN, Sorrondegui M, Alonso M, Sarduy E. 2002. Listeriosis en las especies bovina, ovina y porcina en Cuba. Rev Prod Anim 14: 61-65.

19. Quinn PJ, Markey BK, Carter ME, Donnelly WJ, Leonard EC. 2001. Veterinary microbiology and microbial disease, Blackwell Pub., Oxford, p.72-75.
20. Radostits OM, Gay CC, Hinchcliff KW, Constable PD. 2007. A textbook of the diseases of cattle, horses, sheep, pigs and goats, $10^{\text {th }}$ ed. Saunders, Philadelphia, p. 805-810.

21. Rebhun WC. 1987. Listeriosis. Food Anim Pract 3: 75-83.

22. Rissi DR, Rech RR, Barros RR, Kommers GD, Langohr IM, Pierezan F, Barros CS. 2006. Forma nervosa de listeriose em caprinos. Pesq Vet Bras 26: 14-20.

23. Rissi DR, Glaudia D, Kommers GD, Marco P, Schild ML, Barros CS. 2010. Meningoencefalite por Listeria monocytogenes em ovinos. Pesq Vet Bras 30: 51-56.

24. Sanaa M, Poutrel B, Menard JL, Serieys F. 1993. Risk factors associated with contamination of raw milk by Listeria monocytogenes in dairy farms. J Dairy Sci 76: 28912898.

25. Staric J, Krizanec F, Zadnik T. 2008. Listeria monocytogenes keratoconjunctivitis and uveitis in dairy cattle. Bull Vet Inst Pulawy 52: 351-355.

26. Stöber M. 2005. Listeriosis. In: Medicina interna y cirugía del bovino (Dirksen G, Gründer H, Stöber M ed.), $4^{\circ}$ ed, Interamericana, Buenos Aires, p.1119.

27. Summers BA, Cummings JF, Lahunta A. 1995. Veterinary Neuropathology, Mosby, St. Louis, p. 133-135.

28. Thompson H, Taylor DJ, Philbey AW. 2009. Fatal mesenteric lymphadenitis in cattle caused by Listeria monocytogenes. Vet Rec 164: 17-18.

29. Weinstock D, Horton SB, Rowland PH. 1995. Rapid diagnosis of Listeria monocytogenes by immunohistochemistry in formalin-fixed brain tissue. Vet Pathol 32: 193195.

30. Wesley IV, Larson DJ, Harmon KM, Luchansky JB, Schwartz AR. 2002. A case report of sporadic ovine listerial meningoencephalitis in Iowa an overview of livestock and human cases. J Vet Diagn Invest 14: 314-321. 